\title{
Density estimation in elastic full waveform tomography
}

\author{
Keisuke TERANISHI ${ }^{1}$, Hitoshi MIKADA ${ }^{1}$, Goto TADANORI ${ }^{1}$ and Junichi TAKEKAWA ${ }^{1}$ \\ ${ }^{1}$ Dept. of Civil and Earth Res. Eng., Kyoto University
}

\begin{abstract}
Seismic Full-Waveform Tomography (FWT) method has been used to estimate velocity structure in the subsurface using acoustic wave equation. In elastic FWT, density is usually estimated using an empirical formula or is fixed to a constant value. Almost all elastic FWT studies have ignored the influence of density that could be an important parameter. The objective of this study is to investigate the difficulty of estimating density structure and propose a new approach. In this study, we perform series of numerical simulations in order to investigate the important factor in the inversion of density structure. Our results show that it is difficult to estimate an accurate density model in the density sorely inversion because the density structure is less effective to the waveform, and the density parameter is more strongly related to the Vp parameter than Vs. We propose s new strategy based on the separations of P-wave and S-wave, and of two inversion stages: the first stage to invert Vp and density structures simultaneously, and the second one to invert Vp, Vs and density together. We conclude that this strategy indicates effective way to estimate an accurate density structure.
\end{abstract}

\section{INTRODUCTION}

Seismic full-waveform inversion (FWI) is a useful elastic constant values building method to prospect the subsurface structure. The method estimates the properties in the areas of interest for using the full waveform information, and the importance to use waveform is widely recognized in seismic explorations. The majority problem in elastic FWI is a multiparameter estimation, especially density structure. As wave propagation is influenced by elastic parameter $\mathrm{Vp}, \mathrm{Vs}$, density, it is necessary to include these parameters in modeling and inversion ${ }^{1)}$. However, FWI problem becomes ill-posedness if the density structure is included as a parameter in the application of elastic FWI. So there are a few previous studies estimating the density structure directly. Many elastic FWI studies have been referred to an empirical formula such as Gardner's relationship ${ }^{2)}$ for estimating density structure or ignored density variation because of the difficulty to estimate.

However, density is the useful parameter to characterize the reservoir. So it is necessary to invert density to prospect the accurate subsurface structure. In this paper, we investigate the important factor to estimate the density structure appropriately. Subsequently, we propose a new approach to invert the density structure.

\section{THEORY}

A new inversion strategy was built in 1980s known as full-waveform inversion ${ }^{3)}$. FWI updates the properties of the subsurface parameter from observed data. To calculate the model parameter direction, waveform inversion algorithm seeks to minimize the residual between the forward simulation and the observed seismic data. In general, the modeled seismic wavefields $\mathbf{d}_{\text {cal }}$ is calculated at the receiver points for each source.

$$
\mathbf{d}_{\mathrm{cal}}=\mathbf{S}(\mathbf{m})
$$

where $\mathbf{S}$ represents the non-linear function and $\mathbf{m}$ is model parameter. In elastic FWI, $\mathbf{m}$ represents Vp, Vs and density parameter.

The residual vector is defined as the difference the modeled seismic wavefields and observed seismic data:

$$
\Delta \mathbf{d}=\mathbf{d}_{\text {cal }}-\mathbf{d}_{\text {obs }}
$$

We use the $\mathrm{L}_{2}$ norm based misfit function given by 


$$
\mathbf{E}(\mathbf{m})=\frac{1}{2}\|\Delta \mathbf{d}\|^{2}=\frac{1}{2} \Delta \mathbf{d}^{\mathrm{T}} \Delta \mathbf{d}
$$

where $\mathbf{E}(\mathbf{m})$ is the data misfit function and ${ }^{\mathbf{T}}$ means the matrix transpose. Our purpose is to estimate the optimal model $\mathbf{m}$ which minimize the misfit function $\mathbf{E}(\mathbf{m})$.

The gradient method is obtained the gradient which minimizes the $\mathbf{E}(\mathbf{m})$ by updating model parameter.

$$
\mathbf{m}^{\mathbf{k}+1}=\mathbf{m}^{\mathbf{k}}-\alpha \nabla \mathbf{E}(\mathbf{m})
$$

where the superscripts represent iteration numbers and is a step length which estimated by using parabolic interpolation through three points(Nash, 1979) $)^{4}$. The gradient of the misfit function can be obtained by either taking partial derivatives or using the backpropagation method which are given by Tarantola(1984). We use the backpropagation method and get the gradient direction taking the following form:

$$
\nabla \mathbf{E}(\mathbf{m})=\left[\mathbf{F S}^{-1} \Delta \mathbf{d}\right]
$$

where $\mathbf{F}$ represents the virtual source.

We perform the modeling with a conventional time-domain acoustic finite-difference method discretizing the scalar wave equation and elastic finite-difference method that discretizing the velocity stress formulation of the elastic wave equation on a staggered grid $^{5)}$. Expression for elastic parameter gradient direction have been derived from a second-order elastic wave equation ${ }^{6)}$.

\section{MODEL SETTINGS}

Figure 1 shows model geometry and source-receiver setup and Table 1 shows the corresponding recording geometries and Finite Difference Time Domain (FDTD) parametrizations. The starting models are homogeneous and have constant values of "background". The synthetic noise-free data were calculated using the finite-difference solution of acoustic and elastic wave equations. For the absorbing boundary, acoustic simulation uses Reynolds boundary condition $^{7)}$ and elastic simulation uses Cerjan boundary condition ${ }^{8}$. Source is a ricker wavelet which has $25 \mathrm{~Hz}$ peak frequency. The total of 9 shot gathers were generated at a $120 \mathrm{~m}$ interval and recorded with an interval of $12 \mathrm{~m}$.

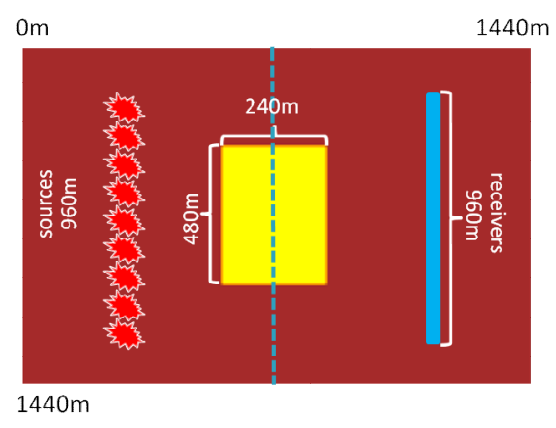

Figure 1: Yellow rectangle represents anomaly, red signs are source settings and blue line represents receiver geometries. Inversion results compare at model center along a blue dashed line.

Table 1: Overview of finite-difference parametrization of models.

\begin{tabular}{|l||l|}
\hline Total X,Z distance & $1440[\mathrm{~m}]$ \\
\hline Grid interval & $\Delta x=6 \mathrm{~m}, \Delta z=6 \mathrm{~m}$ \\
\hline Vp velocity (back ground) & $3000[\mathrm{~m} / \mathrm{s}]$ \\
\hline Vs velocity (back ground) & $1334[\mathrm{~m} / \mathrm{s}]$ \\
\hline Density (back ground) & $2000\left[\mathrm{~kg} / \mathrm{m}^{3}\right]$ \\
\hline Vp velocity (anomaly) & $3200[\mathrm{~m} / \mathrm{s}]$ \\
\hline Vs velocity (anomaly) & $1422[\mathrm{~m} / \mathrm{s}]$ \\
\hline Density (anomaly) & $2300\left[\mathrm{~kg} / \mathrm{m}^{3}\right]$ \\
\hline Time step & $1[\mathrm{~ms}]$ \\
\hline Total sampling time & $1[\mathrm{~s}]$ \\
\hline Peak frequency & $25[\mathrm{~Hz}]$ \\
\hline Source interval & $120[\mathrm{~m}]$ \\
\hline Receiver interval & $12[\mathrm{~m}]$ \\
\hline Total number of sources & 9 \\
\hline Total number of receivers & 80 \\
\hline
\end{tabular}

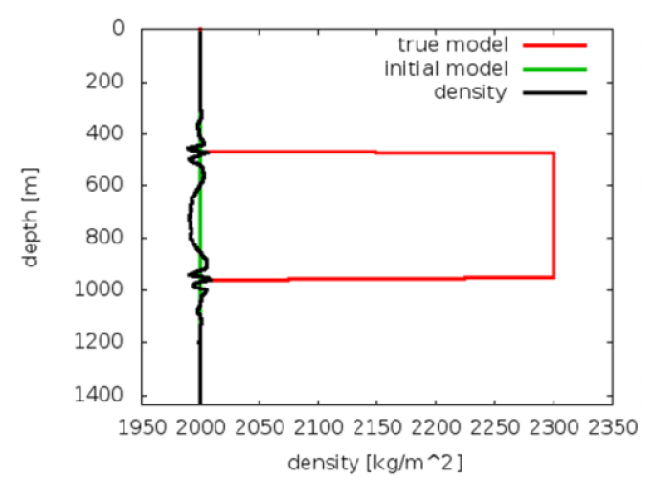

Figure 2: density singly inversion result (density).

\section{RESULTS AND DISCUSSION}

We estimate only density structure to investigate the influenced factor of density estimation. Therefore, $\mathrm{Vp}$ and Vs structures are same as the true model. Figure 2 shows the result of density at the center of the model. True model is denoted by red line, initial model is denoted by green line and 
inversion result is denoted by black line. Residual between true and initial model shot gather is shown in Figure 3.

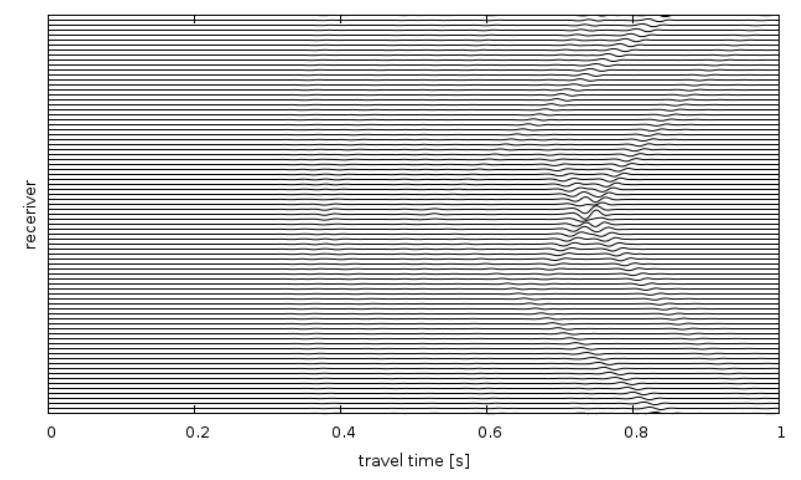

Figure 3: residual shot gather seismogram between true and initial model shot gather.

Figure 2 indicates that density only inversion cannot estimate appropriate density values because there are very small waveform residuals shown the results of Figure 3. So inversion result is failure updating the density parameter. Therefore, estimating density structure singly is difficult and density should be inverted with other parameters.

Next, we tested series of numerical experiments to evaluate other parameter affected density estimation. Strategy (a) inverts $\mathrm{Vp}$ with acoustic inversion before that $\mathrm{Vp}, \mathrm{Vs}$, density estimation in elastic inversion. Strategy (b) inverts a conventional elastic inversion estimating $\mathrm{Vp}, \mathrm{Vs}$, density. These strategy investigate whether $\mathrm{Vp}$ parameter effect density estimation or not.

Figure 4 shows these inversion results of each strategy (a) and (b). From result of (a-1), Vp structure is estimated accurately because acoustic inversion was applied which are used the direct arrivals of P-wave information for updating only $\mathrm{Vp}$ structure. The result of (a-2) is same as result of (b-2) in Vs structure. For density structure, however, (b-3) is more accurate than (a-3). These analyses suggest density structure should be more related to Vp structure and less related to Vs structure.

From the above numerical results strategy (a) and (b), we can propose a new approach that first elastic inversion inverts $\mathrm{Vp}$ and density structure simultaneously with direct arrivals of P-wave and then inverts $\mathrm{Vp}, \mathrm{Vs}$ and density structure simultaneously with direct arrivals of S-wave (strategy (c)).

Figure 5 shows the result of strategy (c). The results of (c-1) (c-3) show that the strategy (c) is effective to estimate the density structure than other strategies (a),(b). The density structure should be inverted using direct arrivals of P-wave. Therefore, inverting $\mathrm{Vp}$ and density structure simultaneously with direct arrivals of $\mathrm{P}$-wave in elastic inversion provides reliable result of density structure.

\section{CONCLUSION}

We presented an implementation of elastic parameter estimation, especially density structure using based on full waveform inversion. First, individual estimation of density structure indicated that it is difficult to invert density structure because of less effectiveness to the waveform. Also this result indicates density structure should be inverted simultaneously with other parameters. So we conducted series of numerical experiments to investigate the effectiveness of the proposed method. From the comparison of combination of acoustic with elastic inversion with conventional elastic inversion, density structure is more influenced by $\mathrm{Vp}$ parameter than Vs parameter. We proposed an approach which firstly estimates $\mathrm{Vp}$ and density structure together by the use of direct P-wave before the estimation of $\mathrm{Vp}$, Vs and density structure by the use of direct $\mathrm{S}$-wave. As a result, $\mathrm{Vp}$ and density simultaneous estimation provides more accurate inversion result. To apply this technique, it is important to separate direct $\mathrm{P}$-wave and S-wave precisely.

\section{REFERENCES}

1)Virieux, J. \& Operto, S., 2009. An overview of full-waveform inversion in exploration geophysics, Geophysics, 74(6), WCC1-WCC26.

2)Gardner, L. and A. Gregory, 1974, Formation velocity and density-the diagnostic basics for stratigraphic traps. Geophysics, 39, 770-780.

3)Tarantola, A., 1984, Inversion of seismic reflection data in the acoustic approximation: Geophysics, 49, 1259-1266.

4)Nash, J.C., 1979, Compact Numerical Methods for Computers: Linear Algebra and Function Minimization, Institute of Physics Publications, Bristol.

5)Virieux, J., 1986, P-SV wave propagation in heterogeneous media Velocity-stress finite difference method, Geophysics, 51, 889-901.

6)Mora, P., 1987, Nonlinear two-dimensional elastic inversion of multi offset seismic data: Geophysics, 52, 1211-1228.

7) Reynolds A.C., 1978, Boundary conditions for the numerical solution of wave propagation problems, Geophysics, 43, 1099-1110. 


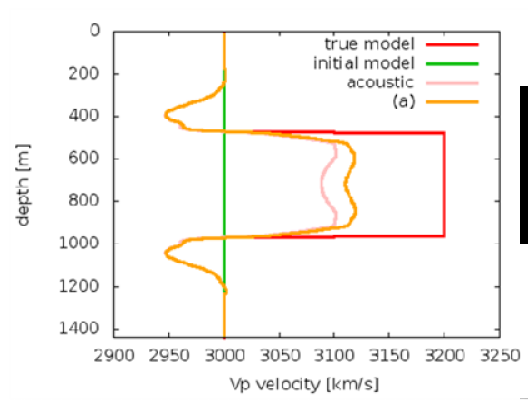

(a-1) Vp structure

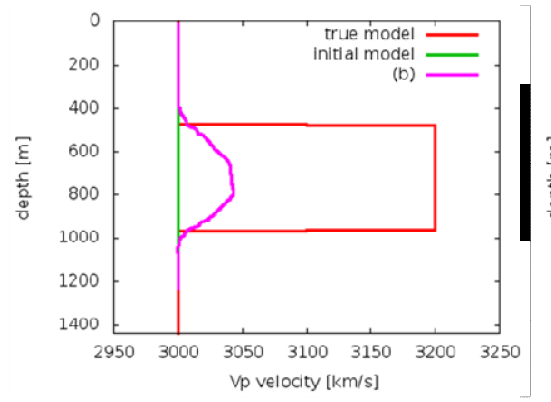

(b-1) Vp structure

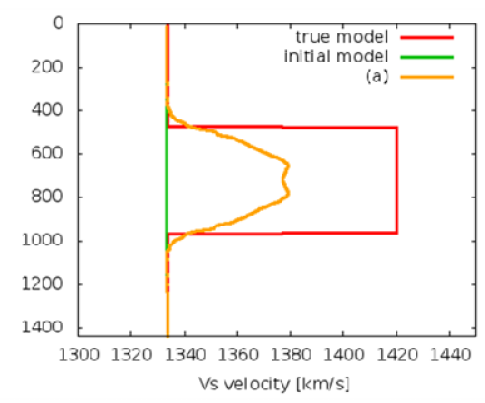

(a-2) Vs structure

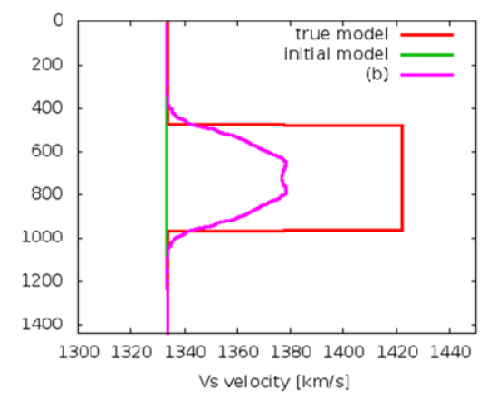

(b-2) Vs structure

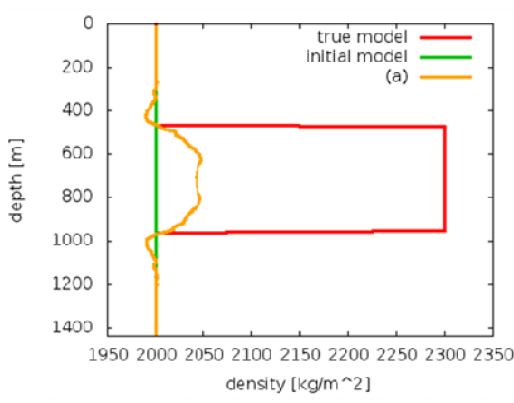

(a-3) density structure

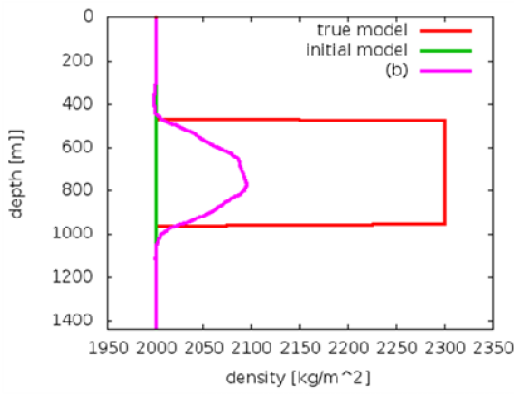

(b-3) density structure

Figure 4: several numerical results. Strategy (a): combination inversion Vp in acoustic inversion and Vp, Vs and density estimation in elastic inversion. Strategy (b): conventional elastic inversion estimating Vp, Vs and density. The first column is Vp results, the second column is Vs results and the third column is density parameter results.

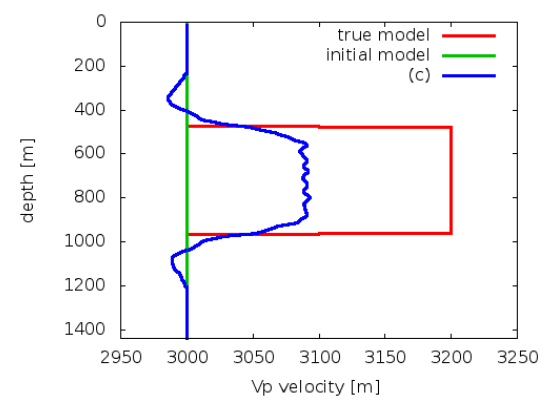

(c-1) Vp structure

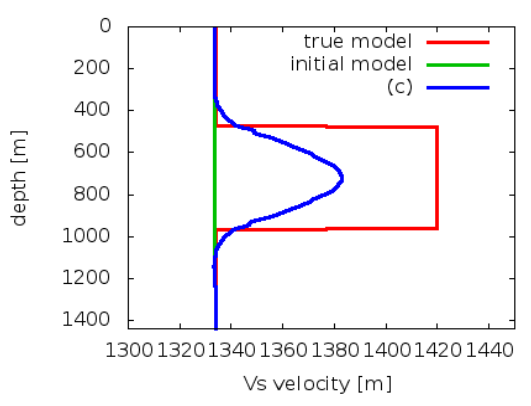

(c-2) Vs structure

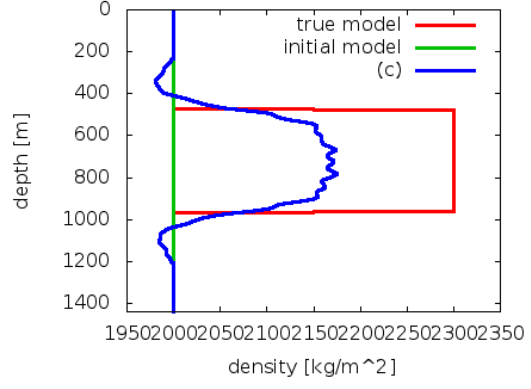

(c-3) density structure

Figure 5: Strategy (c): first estimating Vp, density in elastic inversion with direct arrivals of $\mathrm{P}$-wave and then estimating all parameters in elastic inversion with direct arrivals of S-wave. The first column is Vp results, the second column is Vs results and the third column is density parameter results. 